\title{
Identification of Genetic Alterations in Carrot Cell Lines Using Transposable Elements Belonging to the Tdc1 Family
}

\author{
Yoshio $\mathrm{ITOH}^{*}$ and Yoshihiro OZEKI \\ Department of Biotechnology, Faculty of Technology, Tokyo University of Agriculture and Technology, \\ Koganei, Tokyo 184-8588, Japan \\ *Corresponding author E-mail address: yoss@cc.tuat.ac.jp
}

Received 27 September 2001; accepted 19 November 2001

\begin{abstract}
Genetic alterations, including somaclonal and culture variations, occur during the culture of plant cells. In this study, we established a colored cell line synthesizing anthocyanin as a variant of a colorless cell line. We examined the insertions of transposable elements belonging to the $T d c 1$ family in both the colored and colorless cell lines by inverse polymerase chain reaction (IPCR) using the inverted repeat sequence of $T d c 1$ as a primer. Most amplified DNA fragments differed in length between the colored and colorless cell lines. This reflected the different insertions of transposable elements belonging to the $T d c 1$ family in each of these cell lines, proposing that the movement of $T d c 1$ elements may constitute one of the events causing genetic alteration in somaclonal and culture variations. One of the insertion sites was located in the gene encoding an amino acid sequence similar to $(S)$-reticuline oxidase.
\end{abstract}

Accession numbers: AB071704

Keywords: Carrot, Daucus carota, Inverse PCR, Somaclonal variation, $(S)$-reticuline oxidase, $T d c 1$, Tissue culture, Transposon.

\section{Introduction}

The insertion of a transposable element into a gene often results in the inactivation of that gene, leading to a mutant phenotype. Transposable elements are a valuable genetic tool with which to generate mutants and to isolate the genes tagged by their insertion. New genes for which only a mutant phenotype is known have been isolated by tagging with endogenous transposable elements (for reviews, see Iida et al., 1999; Maes et al., 1999; Walbot, 2000). With recent advances in plant biotechnology and the production of transgenic plants, the $A c$ and $E n / S p m$ transposable elements from maize have been introduced into heterologous plants to tag useful and novel genes (for reviews, see Osborne and Baker, 1995; Kunze et al., 1997; Maes et al., 1999). Similar to tagging tools for class II transposable elements whose transposition intermediates are DNA such as $A c$ and $E n / S p m$ superfamily elements, class I elements, which transpose via RNA intermediates, have been used as genetic tools to disrupt and identify new genes in both the original host plant and transgenic plants (Okamoto and Hirochika, 2000; Kumar and Hirochika, 2001).

In suspension-cultured cells of carrot (Daucus carota), we identified an $\mathrm{En} / \mathrm{Spm}$-like transposable element, $T d c 1$, present in the 5 ' flanking region of a phenylalanine ammonia-lyase gene ( $g D c P A L 1)$, which is induced by transferring the cells of carrot suspension cultures into fresh liquid medium (the transfer or dilution effect) (Ozeki et al., 1997). This particular cell line has been subcultured continuously for 12 years. The original integration of $T d c 1$ into $g D c P A L 1$ did not occur during the first four years of subculture. $T d c 1$ was first detected in gDCPAL1 genomic clones of a genomic library made from the same cultured cell line after seven years of subculture. After 12 years of subculture, about $5-10 \%$ of the cells had $T d c 1$ inserted into the gDCPAL1 gene. During the first 4-7 years of subculture, $T d c 1$ may have inserted into $g D c P A L 1$ in one (or more) cell(s) of ca. $10^{9}$ cells of one batch culture. The insertion of $T d c 1$ into the $g D c P A L 1$ gene reduced the activity of the gene's promoter and the inducibility of $g D c P A L 1$ by transfer-dilution. Consequently, a selective advantage was con- 
ferred upon cells with an inactivated $P A L$ gene under conditions of undifferentiated growth. Therefore, populations of cells with $g D c P A L 1$ harboring $T d c 1$ in batch cultures of this line increased over the last five years of culture (Ozeki et al., 1997). These results indicate that the transposition of $T d c 1$ is one of the mechanisms causing somaclonal and culture variations in cultured carrot cells.

Two types of alterations may play important roles in the mechanisms causing somaclonal and culture variations in cultured plant cells. These are genetic alteration, which is defined as the stable modification of a genomic DNA sequence, and epigenetic alteration, which is defined as a modification in the structure, organization, or regulation of the genotype, and can be highly transmissible under stable culture conditions. However, very few data are available on these mechanisms.

In this study, we established from a colorless carrot cell line a variant colored cell line synthesizing anthocyanin. As an initial step in identifying how much genetic alteration occurs between a parent cell line and a culture variant cell line in plants, we used inverse polymerase chain reaction (IPCR) to identify the different insertions of transposable elements belonging to the $T d c 1$ family in these two cell lines.

\section{Materials and Methods}

Establishment of the colored anthocyanin-synthesizing cell line and the undifferentiated colorless carrot cell line

In 1986, a suspension-cultured carrot cell line was established from a carrot hypocotyl in liquid modified Lin and Staba medium (Lin and Staba, 1961), as described by Fujimura and Komamine (1975). At the time of initiation of cell division from the hypocotyl in liquid medium, a red cell aggregate was observed among the many colorless (yellow) cell aggregates. Cell aggregates were poured onto solid medium containing $0.8 \%$ agar, and the red and yellow cell aggregates were independently picked by hand and inoculated onto fresh solid medium. After the calli had grown, small amounts of the redcolored part of the callus were picked manually using forceps and inoculated onto fresh medium (the "cell aggregate cloning method" reported by Yamamoto et al., 1982). At monthly subculturing, the cell aggregate cloning procedure was repeated for more than seven years, and a colored cell line synthesizing anthocyanin in medium containing 2,4 - dichlorophenoxyacetic acid (2,4-D) was established (Fig. 1A). The colorless cell line was established from a yellow cell aggregate without selection (Fig. 1B).

Extraction of genomic DNA from carrot cells, and IPCR

Genomic DNA was prepared from carrot cells of both cell lines using a previously described cetyltrimethyl-ammonium bromide (CTAB) method (Ozeki et al., 1993). Genomic DNA (100 ng) was digested with six-base - recognizing restriction endonucleases, e.g., BamHI, HindIII, XbaI, or XhoI. After phenol extraction and ethanol precipitation, digested DNA was circularized by self-ligation with the TaKaRa Ligation Kit version II (TaKaRa Biochemicals, Shiga, Japan), and ethanol precipitated. Using circularized genomic DNA (50 ng) as template, IPCR was performed in $40 \mu 1 \mathrm{LA}$ - PCR mixture (TaKaRa Biochemicals), which was prepared according to the supplier's recommendations and contained 4 pmol of one primer, 5'TCGCGTTTTCTTGTAGTG-3', corresponding to the complementary sequence of the terminal inverted repeat of Tdc1 (Ozeki et al., 1997). The mixture was incubated for $1 \mathrm{~min}$ at $98^{\circ} \mathrm{C}$, and then 1 unit of TaKaRa LA-Taq DNA polymerase was added. Forty amplification cycles of PCR were performed $\left(30 \mathrm{~s}\right.$ at $92{ }^{\circ} \mathrm{C} ; 1 \mathrm{~min}$ at $55^{\circ} \mathrm{C} ; 20 \mathrm{~min}$ at $68^{\circ} \mathrm{C}$ ). After the IPCR, the amplified fragments were analyzed on $0.8 \%$ agarose gels. DNA fragments were purified by GeneClean ${ }^{\text {Th }}$ (BIO101 Inc., CA) for use as probes in northern analysis and for plaque hybridization with a carrot cDNA library.

\section{Northern analysis, cDNA cloning, and nucleotide sequencing}

Total RNA was extracted from cultured carrot cells, purified to poly $\left(\mathrm{A}^{+}\right) \mathrm{RNA}$, and northern blotted as previously described (Ozeki et al., 1990). Cells other than those of the colored cell line (lane 1, Figs. 3 and 4) and the colorless cell line (lane $\mathbf{3}$, Figs. 3 and 4) were prepared as follows. Cells of the embryogenic line (lane 2, Figs. 3 and 4) were derived from hypocotyl explants using the method of Osuga et al. (1999). Cells from a different carrot suspension-culture cell line grown in liquid medium containing $2,4-\mathrm{D}$ were fractionated by sieving and density-gradient centrifugation in $12 \%$ Ficoll. Cells floating on the Ficoll solution were then transferred to liquid medium without 2,4-D and cultured for seven days to induce anthocyanin synthesis (lane 4, Figs. 3 and 4) (Ozeki and Komamine, 1981). Cells were induced to synthesize anthocyanin in liquid medium without 2,4-D for six days, after which the accumulation of anthocyanin was suppressed by the addition of 2,4- D $\left(5 \times 10^{-7}\right.$ $\mathrm{M})$ to the medium, and cells were cultured for one 


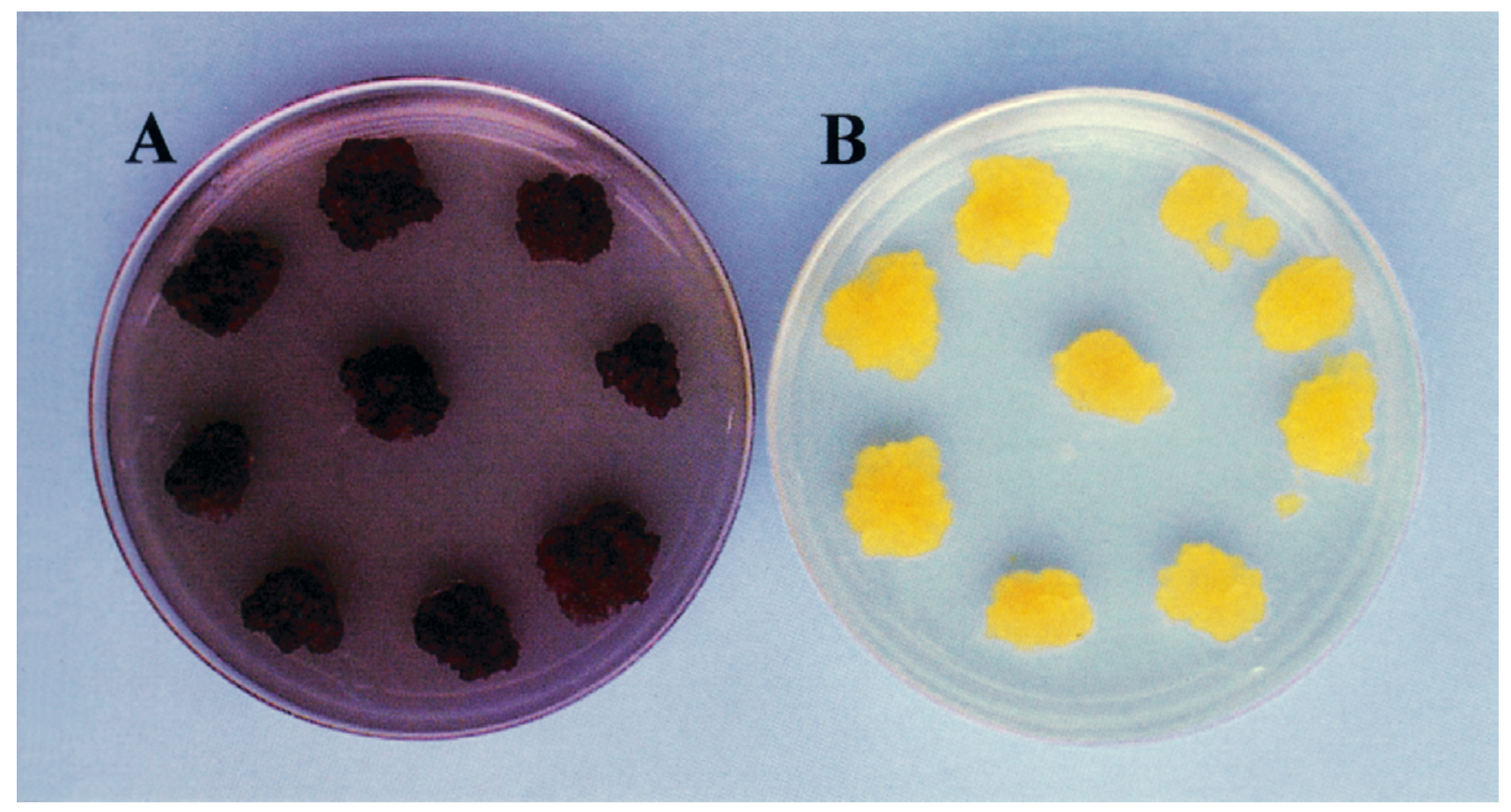

Fig. 1 Carrot callus of the colored cell line that synthesizes anthocyanin (A) and the colorless cell line (B) on medium containing 2,4-D. 
more day (lane 5, Figs. 3 and 4). Another carrot suspension-culture cell line was freshly established and subcultured within one year of its establishment in liquid medium containing 2,4-D. Cells of this line were fractionated by density-gradient centrifugation in $18 \%$ Ficoll, then the cells precipitated on the Ficoll solution were transferred to liquid medium without 2,4-D to induce embryogenesis (Fujimura and Komamine, 1975). Cells were cultured for five days at the globular stage (lane $\mathbf{6}$, Figs. 3 and 4), and cultured for eight days at the torpedo stage (lane 7, Figs. 3 and 4).

A cDNA library was prepared from poly $\left(\mathrm{A}^{+}\right)$ RNA derived from anthocyanin-synthesizing cells using a $\lambda$ ZAPII vector (Stratagene Co. Ltd., CA) according to the supplier's manual. The method used to screen positive clones has been reported previously (Ozeki et al., 1990). Plasmids containing the cDNA were prepared using helper phage according to the supplier's recommendations, and the nucleotide sequence of the cDNA was determined by preparation of serial deletion clones using Exonuclease III digestion followed by treatment with mung-bean nuclease (Ozeki et al., 1990).

\section{Results and Discussion}

Establishment of the colored carrot cell line synthesizing anthocyanin with undifferentiated growth in medium containing $2,4-D$

In 1986, we established a colored cell line in which anthocyanin synthesis occurs with active cell division in medium containing 2,4-D (Fig. 1A) Since then, anthocyanin has been stably synthesized by these cells and the callus has grown well with monthly subculture for more than 12 years. Other researchers have reported the establishment of anthocyanin-synthesizing cell lines of $D$. carota from cultivars other than that used here, with undifferentiated growth in medium containing auxin (Sugano and Hayashi, 1967; Alfermann and Reinhard, 1971; Ibrahim et al., 1971; Schmitz and Seitz, 1972; Dougall et al., 1980). Using D. carota cv. "Kurodagosun", we sometimes found anthocyanin-synthesizing aggregates just after the initiation of cell division from the hypocotyl in medium containing $2,4-$ D. However, in most cases, synthesis of anthocyanin was unstable and the cells synthesizing and accumulating anthocyanin disappeared at an early stage during the establishment of the cell lines (Ozeki, unpublished data). In normal cultured cell lines of $D$. carota cv. "Kurodagosun" maintained in medium containing $2,4-\mathrm{D}$, anthocyanin synthesis only occurs in cells fractionated by sieving and Ficoll density-gradient centrifugation and then transferred to medium without $2,4-\mathrm{D}$, which induces "metabolic differentiation" (Ozeki and Komaminc, 1981). However, when 2,4-D is added to the medium, anthocyanin synthesis is completely suppressed and undifferentiated growth occurs (Ozeki and Komamine, 1981). In numerous experiments we have undertaken to establish carrot cell cultures, the cell line reported here was the only instance in which a colored cell line was established that synthesizes anthocyanin during active cell division in medium containing $2,4-\mathrm{D}$. In this cell line, the genes for enzymes involved in anthocyanin synthesis and the MYB-related genes, which may regulate the expression of the genes for the enzymes involved in anthocyanin synthesis, were expressed continuously in medium containing $2,4-\mathrm{D}$ (data not shown).

Detection and amplification by IPCR of DNA fragments containing the sequences flanking the insertion sites of transposable elements of the $T d c 1$ family in the genomic DNA of the colored and colorless cell lines

Recently, several methods have been established to identify genes disrupted by the insertion of transposable elements, such as transposon display (TD, Van den Broeck et al., 1998), amplification of insertion mutagenized sites (AIMS, Frey et al., 1998), transposon insertion display (TID, Yephremov and Saedler, 2000), and simplified transposon display (STD, Fukuda-Tanaka et al., 2001). These are powerful methods with which to identify transposon-flanking sequences in plant species that have more than a hundred copies of a transposable element in their genomes. However, to achieve high - resolution in the detection and discrimination of more than a hundred DNA fragments that are adjacent to transposon-flanking sequences, the amplified products must be displayed on an acrylamide sequencing gel under denaturing conditions. Because the amounts of amplified products are very small, they must be visualized with $\left[{ }^{32} \mathrm{P}\right]$-labeling or fluorescent labeling, and the products should be shorter than several hundred base pairs. Therefore, it is unlikely that the amplified fragments will contain sequences of protein coding regions, because the amplified fragments were too short. The bands of interest are eluted from the gel and amplified again by PCR, and a genomic library is screened using the reamplified fragments as probes to isolate the sequences of any coding regions neighboring the fragments. In contrast, IPCR is a classical method (Tsuei et al., 1994; Souer et al., 1995), easy but limited in application to the detection of transposon-flanking sequences (Hui et al., 
1998). However, if the terminal inverted repeats of the transposable elements are long enough to act as primer sequences and the copy number of the elements in the genome is not high, IPCR is a convenient and powerful method with which to isolate, on agarose gel, fragments longer than several kilobases containing the transposon-flanking sequences. These amplification products are used directly for northern hybridization and cDNA screening. $T d c 1$ has a terminal inverted repeat

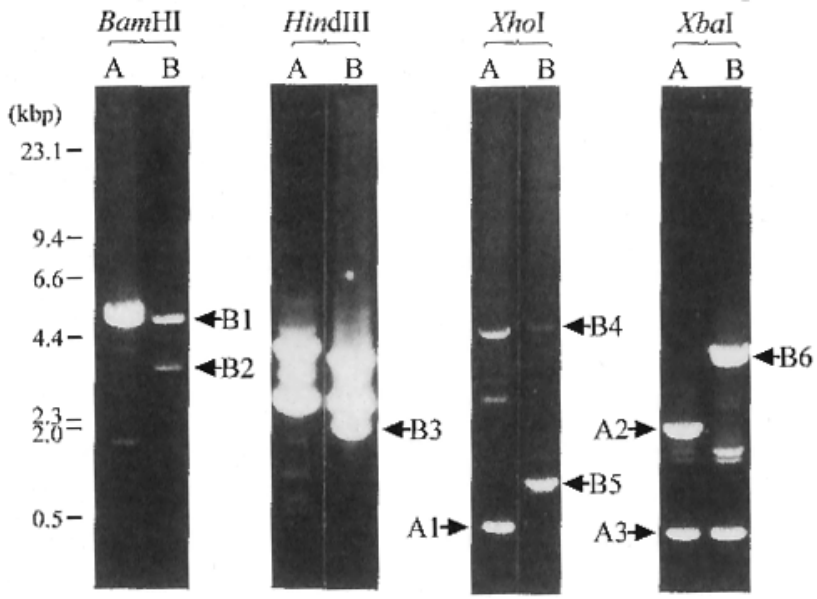

sequence of 18 bp and its copy number is about 30 to 40 per genome (Ozeki et al., 1997), making it a suitable target for IPCR.

Genomic DNA digested with BamHI, HindIII, $X h o I$, or $X b a \mathrm{I}$, and self-ligated to form circularized DNA, was used as the template for IPCR, with a single primer corresponding to the sequence of the $T d c 1$ terminal inverted repeat. Different patterns of amplified fragments were observed for the colored and colorless lines (Fig. 2). These results indicate

Fig. 2 DNA fragments amplified by IPCR from the colorless and colored lines. Genomic DNA was prepared from the callus of the colorless cell line (A) and the colored cell line that synthesizes anthocyanin (B), and was digested with Bam HI, HindIII, $X h o \mathrm{I}$, or $X b a \mathrm{I}$ followed by self-ligation. DNA thus circularized was used as the template for IPCR with a single primer corresponding to the sequence of the $T d c 1$ terminal inverted repeat. Amplified fragments were separated by electrophoresis on $0.8 \%$ agarose, and visualized with ethidium bromide staining.

A1
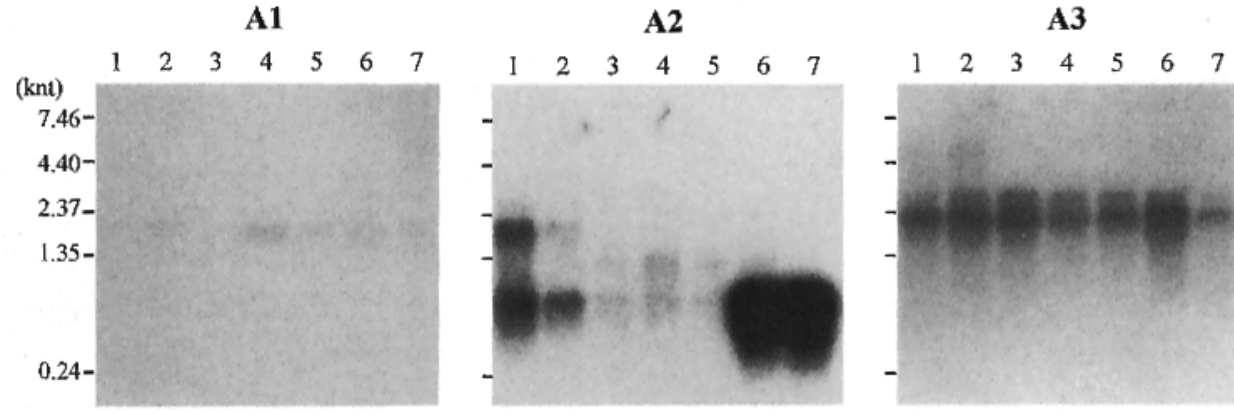

B1

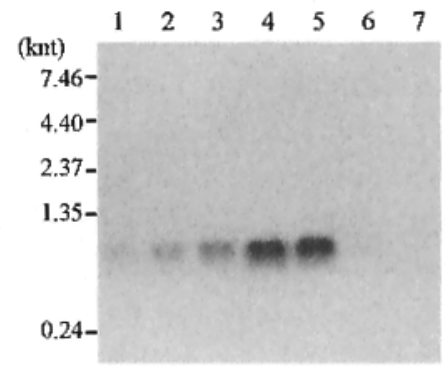

B2

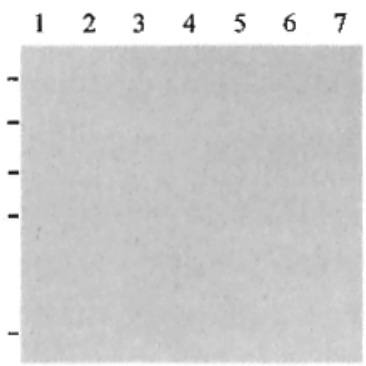

B3

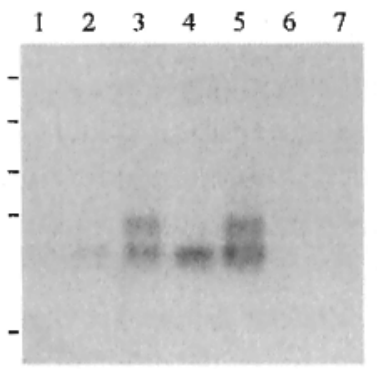

B4

B5

B6
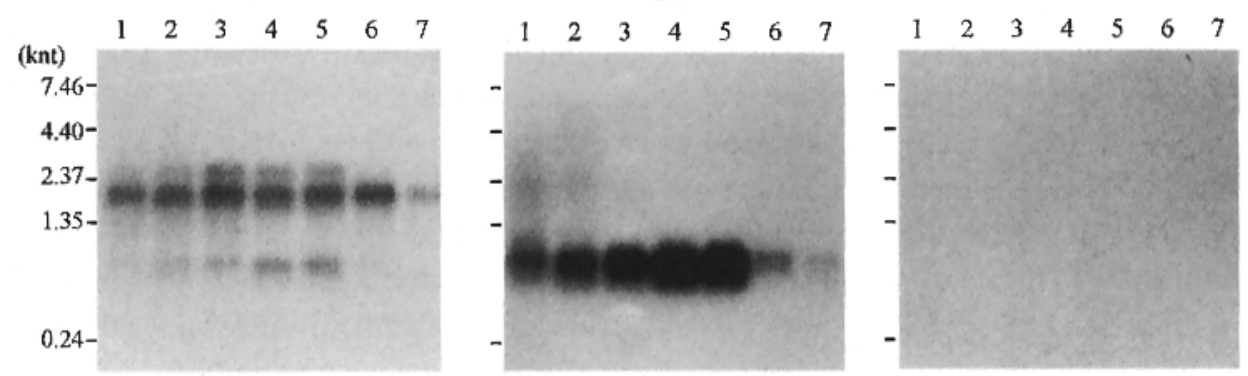
that the transposable elements belonging to the $T d c 1$ family had inserted into different positions in the genomes of the colored and colorless lines. Three fragments, A1, A2, and A3, were specifically amplified from the genomic DNA of the colorless cell line, and six fragments, B1, B2, B3, B4, B5, and B6, were specifically amplified from the genomic DNA of the anthocyanin-synthesizing cell line (Fig. 2). These nine fragments were purified from the agarose gel and labeled with $\left[{ }^{32} \mathrm{P}\right]-\mathrm{dCTP}$ for use as probes in northern hybridization analysis (Fig. 3). Of the nine fragments, seven produced signals, indicating that seven of the nine fragments contained transcribed sequences. If the transposable elements of the $T d c 1$ family inserted with equal probability into any position in the carrot genome, the elements are more likely to be found in spacer regions between genes than in or close to genes, and consequently more fragments would produce no signal with northern hybridization. It is not known whether transposable elements insert preferentially into particular positions in genomes or what such a preferred nucleotide sequence in the genome might be, but our results indicate that the insertion sites of several elements belonging to the $T d c 1$ family are located in or close to genes.

Probes prepared from A1, A2, and A3, derived from the colorless cell line, suggest that the $T d c$ transposable elements had inserted into or close to the genes encoded by the sequences in the fragments A1, A2, and A3. However, the gene encoded by the sequence in the $\mathrm{A} 3$ fragment was expressed in the colorless cells (Fig. 3, lane 3). In contrast, the fragments B1, B3, B4, and B5 were derived from the colored anthocyanin-synthesizing cell line, but genes encoded by the sequences in all these fragments were expressed in anthocyanin-synthesizing cells (Fig. 3, lane 1). These results suggest two alternative interpretations. It is possible that, when the element inserted close to the genes hybridized with the probes, gene expression was not affected. Alternatively, the element may have inserted into one gene of a multi-gene family in the carrot genome. These possibilities remain to be examined. However, we here observed the differentiationspecific expression of genes encoded by the se- quences in some fragments.

Isolation and characterization of a cDNA encoding a sequence of the A1 fragment

Of the seven transcribed fragments amplified by IPCR, the genes encoded by the A3 and B4 fragments were transcribed continuously in cells of all cultured lines examined here, irrespective of the differentiation state of the cells (Fig. 3). However, some of the other fragments, e.g., A1, A2, B3, and B5, exhibited different expression patterns in cells in different differentiation states. The gene encoded by the sequence in the $\mathrm{A} 1$ fragment was not expressed in colorless cells in medium containing 2,4D (Fig. 3, lane 3), but was expressed in cells in which anthocyanin synthesis or embryogenesis was induced (Fig. 3, lanes 1, 4-7). Using the A1 fragment as a probe, we screened a cDNA library prepared from carrot cells synthesizing anthocyanin, and isolated one positive clone. The CDNA in the positive clone, designated cA1 cDNA, exhibited the same expression pattern when used as a probe in northern analysis as did the A1 fragment (Fig. 4). The nucleotide sequence of cA1 cDNA revealed

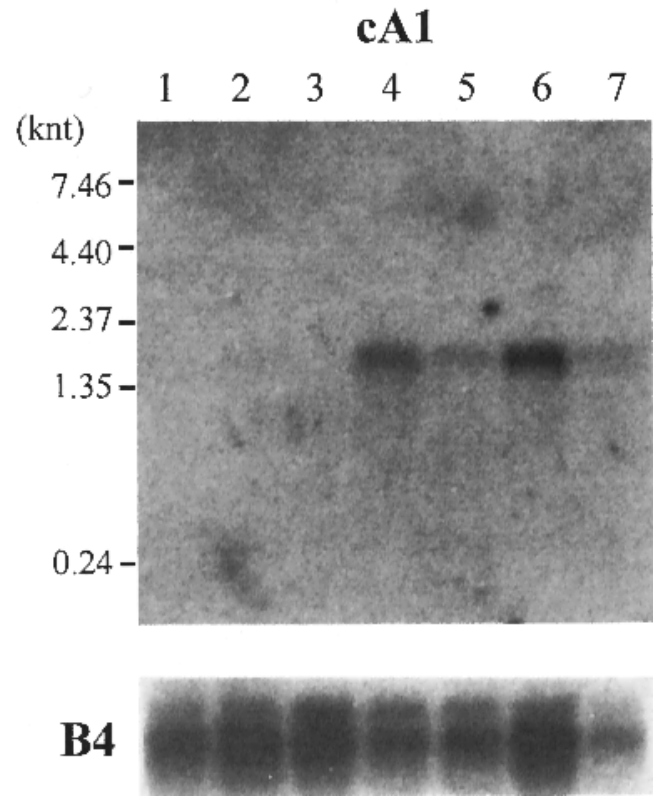

Fig. 4 Northern hybridization with cA1 cDNA used as probe. Poly $\left(\mathrm{A}^{+}\right)$RNA was prepared as for Fig. 3. The B4 fragment was used to control for the amount of RNA loaded into each lane.

Fig. 3 Northern hybridization using IPCR - amplified fragments as probes. Poly $\left(\mathrm{A}^{+}\right)$RNA was prepared from the following cells: 1. the callus of the colored cell line, as shown in Fig. 1A; 2. embryogenic callus; 3. callus of the colorless cell line, as shown in Fig. 1B; 4. anthocyanin-synthesizing cells in liquid medium lacking 2,4-D (Ozeki and Komamine, 1981); 5. anthocyanin-synthesizing cells one day after the addition of 2,4-D (5 $\times 10^{-7}$ M) to the medium; 6. cells at the globular stage; 7. cells at the torpedo stage. (A1), (A2), (A3), (B1), (B2), (B3), (B4), (B5), and (B6) were prepared from DNA fragments by IPCR, as shown in Fig. 2, and used as hybridization probes. 
CAI

Arabidopsis thaliana California poppy

CA1

Arabidopsis thaliana California poppy

CA1

Arabidopsis thaliana California poppy

CA1

Arabidopsis thaliana California poppy

CA1

Arabidopsis thaliana California poppy

cA1

Arabidopsis thaliana California poppy

CA1

Arabidopsis thaliana California poppy

CA1

Arabidopsis thaliana California poppy

CAI

Arabidopsis thaliana California poppy

CA1

Arabidopsis thaliana California poppy
1 -

1 MGNSKPIFTISCIIVSVUYFSFYCITPISSSASIQD-QFINOVKRNTHVSFPLEKIIFTP 1 MEN-KT-E-I-D.---FESLS-IFLSLINCALGGNDLLSCLTENG-VRNH---TVESA

32 A-NAS-YNPIILINIQN-IR FNTSGTRKRIAIVIPIEETCICTVIYOARKNSMNVRTRGE 88 60 AKNVSIFNQVIESTAQN-ICELAKSMPKRGFIERPIHQSQVQASIICSKKLGIHFRVRSG 118 45 DSDSD-ENRFIFISIQNPI-EONSLISKFSAIIIPGSKEELSNIIROIRKGSWTIFIRSG 102

89 GHDFEGVSYTAEV--PFVLLDMINENRVNIDLKTSTAWVQSGISLGEFYYRISOKSDVIA 146 119 GHDFEALSYVSRIEKPFITIDLSKLKOINVDIE SNSAWVOFGATLGELYYRIAEKSKIHG 178 103 GHSYEGLSYTSDT--PFII IDLMNLNRVSIDLESETAWVESGSTLGELYYAITESSSKLG 160

147 FPAGLLSSVELTGLIEGGGYGMLKRKYALAADNILDARIVDYNGKILDFKSMGEDLFWAI 206 179 FPAGLOISVEIGGYMTGGGYGILMRKYGLAGDNVLDVKMVDANGKILDFAAMGEDLFWAI 238 161 FTAGWOPVGTGGHISGGGEGMMSRKYGLAADNVVDAILIDANGA ILDRQAMGEDVEWAI 220

207 RGGDPASERVVLELKLOLVPVPKSVIYFAVORTLEON-GSAIFOKWCAIAANVEPRDLDV 265 239 RGGGGASEGIVLAWK IKLVPVPKTVIVETVTKTLEQDARLKTISKWOQISSKI IEE-IHI 297 221 RGGGGGVWGAIYAKIKIIPVPEKVTVERVTKNVAIDEATSILHKWOEVAEELEE-DFTL 279

266 RVVVDTITSNSSPRQDKKTVRFVFQCLYLGKIDTLLPIMQKY-FPELGLVRDDCTETSWI 324 298 RVVLRAAG-NDGN----KTVTMIYLGQELGEKGTLLKVMEKA-FPELGLTOKDCTEMSWI 351 280 -SVLGCA-D

325 KMAPMFS-G-FPVGTDPMILIN-KTAIPRNSV---KIKSSFTTQPISIEGINGIWDLWIK 378 352 E-AAIFH-GGFPTGSPIE ILIQLKSFIGK-DY-DKAISDEVKEPIPVIGLKGIFKRII- 405 330 ESHA-YLAG-LETVSQ---LNNRF-I-KFDERAFKTKVDITKEPLPSKAFYGLIERISK 381

379 QPVQ-TIL IQYTPFGGIMNE EAESAIPFPHRPEVIYMINMAVTLAONEEAT---LOWIN 433 406 EGNT-THLN-hTEYGGMMSKIPESAIPFPHRNGTIFKIIYYANWIFND-KTSSRKINWIK 462 382 EPNGEIALN---GFGGQMSKISSDFTPFPHRSGTRLMVEYIVAWNCSEOKKKTEELDWIE 438

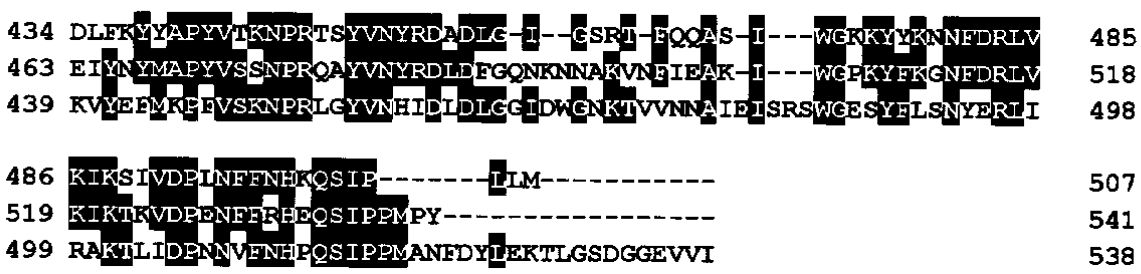

Fig. 5 Amino acid sequence of the open reading frame of cA1 cDNA deduced from the nucleotide sequence (accession number AB071704), and its homology to $(S)$ - reticuline oxidase identified from a cDNA library of elicited cell-suspension cultures of California poppy (Dittrich and Kutchan, 1991), compared with one representative amino acid sequence (accession number AB011475) from the 12 Arabidopsis genes with sequence similarity to $(S)$-reticuline oxidase (identified by the Arabidopsis Genome Initiative, 2000). Identical amino acids are shown on a black background. Hyphens indicate gaps in the alignment. Sequence similarities in the ORFs of $\mathrm{CA} 1$ and the Arabidopsis protein, and between cA1 and the California poppy protein, are $45.2 \%$ and $36.5 \%$, respectively.

that its deduced amino acid sequence has some similarity to $(S)$-reticuline oxidase (Fig. 5). A cDNA encoding $(S)$-reticuline oxidase was first isolated from a cDNA library constructed from elicited cell-suspension cultures of California poppy (Eschscholtzia californica) (Dittrich and Kutchan, 1991). (S)-Reticuline oxidase, which is called the berberine bridge enzyme $[(S)$-reticuline:oxygen oxidoreductase (methylene-bridgeforming), EC 1.5.3.9], is a vesicular enzyme that catalyzes the formation of the berberine bridgehead carbon of $(S)$-scoulerine from the $N$-methyl carbon of $(S)$ - reticuline (Kutchan and Dittrich, 1995). $(S)$ Scoulerine is the biosynthetic precursor of a multi- tude of species-specific protopine, protoberberine, and benzophenanthridine alkaloids. The Arabidopsis Genome Project identified 12 Arabidopsis genes with sequence similarity to $(S)$-reticuline oxidase, suggesting that Arabidopsis may produce alkaloids (Arabidopsis Genome Initiative, 2000). However, the amino acid sequence encoded by cA1 cDNA is similar to the sequence from residues 100 252 of California poppy $(S)$-reticuline oxidase, but there is a remarkable level of dissimilarity in the remaining sequence. Carrot may have more than five genes that can hybridize with cA1 cDNA (Fig. 6). It is possible that a gene other than $\mathrm{CA} 1$ in the carrot genome encodes the real $(S)$-reticuline oxi- 


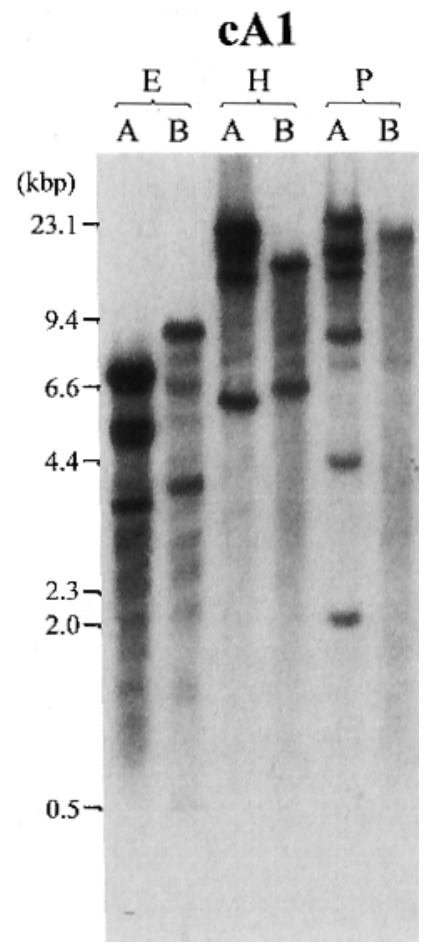

Fig. 6 Comparison of restriction fragment patterns of the colorless cell line (A) and the colored cell line that synthesizes anthocyanin (B). Genomic DNA was extracted from cells of each line and digested with EcoRI (E), HindIII (H), and PstI (P). Southern hybridization was performed using cA1 cDNA as the probe.

dase protein, or an oxidoreductase catalyzing another substrate. We have not yet determined whether cA1 cDNA really encodes carrot $(S)$-reticuline oxidase. Future biochemical experiments will confirm the enzymatic activity of the protein encoded by cA 1 cDNA.

The genomic structure of the $(S)$-reticuline oxidase-like gene family is very different in the colorless cells and the colored cells (Fig. 6). Two completely different patterns of bands were detected by Southern hybridization in the two lines (Fig. 6). This indicates that considerable culture variation has occurred to alter the genomic structure around the $(S)$-reticuline oxidase-like gene family during the initiation of cell division and the subsequent selection of cell aggregates and successive subculturing. Alterations in gene structure, such as rearrangement, recombination, and deletion, may have occurred around the $(S)$-reticuline oxidase-like genes of carrot. Conversely, one of the mechanisms altering the genome structure around the $(S)$-reticuline oxidase-like genes may have been the insertion of a transposable element belonging to the $T d c 1$ family into one or more of the $(S)$ - reticuline oxidase-like gene(s).

Our results show that transposable elements of the
$T d c 1$ family inserted into several differing positions in the genomes of a colored culture variant cell line and the colorless parent cell line in carrot. Genetic alteration may be attributed to several events that cause somaclonal and culture variations in cultured plant cells. It is proposed that the movement of transposable elements may constitute one of the events causing genetic alteration in somaclonal and culture variations.

\section{Acknowledgements}

This work was supported by Grants-in-Aid for Scientific Research from the Ministry of Education, Culture, Sports, Science and Technology of Japan and by the Japan Health Sciences Foundation.

\section{References}

Alfermann, W., Reinhard, E., 1971. Isolierung anthocyanhaltiger und anthocyanfreier Gewebestamme von Daucus carota: Einfluss von Auxinen auf die Anthocyanbildung. Experientia, 27: 353-354.

Arabidopsis Genome Initiative, 2000. Analysis of the genome sequence of the flowering plant Arabidopsis thaliana. Nature, 408: 796-815.

Dittrich, H., Kutchan, T. M., 1991. Molecular cloning, expression, and induction of berberine bridge enzyme, an enzyme essential to the formation of benzophenanthridine alkaloids in the response of plants to pathogenic attack. Proc. Natl. Acad. Sci. U. S. A., 88: $9969-$ 9973.

Dougall, D. K., Johnson, J. M., Whitten, G. H., 1980. A clonal analysis of anthocyanin accumulation by cell cultures of wild carrot. Planta, 149: 292-297.

Frey, M., Chomet, P., Stettner, C., Gierl, A., 1998. A general method for gene isolation in tagging approaches: amplification of insertion mutagenised sites (AIMS). Plant J., 13: $717-721$.

Fujimura, T., Komamine, A., 1975. Effects of various growth regulators on embryogenesis in a carrot suspension culture. Plant Sci. Lett., 5: 359-364.

Fukuda-Tanaka, S., Inagaki, Y., Yamaguchi, T., Iida, S., 2001. Simplified transposon display (STD): a new procedure for isolation of a gene tagged by a transposable element belonging to the TpnI family in the Japanese morning glory. Plant Biotechnol., 18: 143149.

Hui, E. K., Wang, P. C., Lo, S. J., 1998. Strategies for cloning unknown cellular flanking DNA sequences from foreign integrants. Cell Mol. Life Sci., 54: $1403^{-}$ 1411.

Ibrahim, R. K., Thakur, M. L., Permanand, B., 1971. Formation of anthocyanins in callus tissue cultures. Lloydia, 34: 175-182.

Iida, S., Hoshino, A., Johzuka-Hisatomi, Y., Habu, Y., Inagaki, T,, 1999. Floricultural traits and transposable elements in the Japanese and common morning glories. Ann. N. Y. Acad. Sci., 870: 265-274.

Kumar, A., Hirochika, H., 2001. Applications of retrotran- 
sposons as genetic tools in plant biology. Trends Plant Sci., 6: 127-134.

Kunze, R., Saedler, H., Lonnig, W. E., 1997. Plant transposable elements. Adv. Bot. Res., 27: 331-470.

Kutchan, T. M., Dittrich, H., 1995. Characterization and mechanism of the berberine bridge enzyme, a covalently flavinylated oxidase of benzophenanthridine alkaloid biosynthesis in plants. J. Biol. Chem., 270: $24475-$ 24481.

Lin, M., Staba, J., 1961. Peppermint and spearmint tissue cultures. I. Callus formation and submerged culture. Lloydia, 24: 139-145.

Maes, T., De Keukeleire, P., Gerats, T., 1999. Plant tagnology. Trends Plant Sci., 4: 90-96.

Okamoto, H., Hirochika, H., 2000. Efficient insertion mutagenesis of Arabidopsis by tissue culture-induced activation of the tobacco retrotransposon Tto1. Plant J., 23: $291-304$.

Osborne, B. I., Baker, B., 1995. Movers and shakers: maize transposons as tools for analyzing other plant genomes. Curr. Opin. Cell Biol., 7: 406-413.

Osuga, K., Masuda, H., Komamine, A., 1999. Synchronization of somatic embryogenesis at high frequency using carrot suspension cultures: model systems and application in plant development. Methods Cell Sci., 21: $129-140$.

Ozeki, Y., Komamine, A., 1981. Induction of anthocyanin synthesis in relation to embryogenesis in a carrot suspension culture: correlation of metabolic differentiation with morphological differentiation. Physiol. Plant., 53: 570-577.

Ozeki, Y., Davies, E., Takeda, J., 1993. Structure and expression of chalcone synthase gene in carrot suspension-cultured cells regulated by 2,4-D. Plant Cell Physiol., 34: 1029-1037.

Ozeki, Y., Davies, E., Takeda, J., 1997. Plant cell culture variation during long-term subculturing caused by insertion of a transposable element in a phenylalanine ammonia-lyase (PAL) gene. Mol. Gen. Genet., 254:
$407-416$.

Ozeki, Y., Matsui, K., Sakuta, M., Matsuoka, M., Ohashi, Y., Kano-Murakami, Y., Yamamoto, N., Tanaka, Y., 1990. Differential regulation of phenylalanine ammonia -lyase genes during anthocyanin synthesis and by transfer effect in carrot cell suspension cultures. Physiol. Plant., 80: 379-387.

Schmitz, M., Seitz, U., 1972. Hemmung der Anthocyansynthese durch Gibberellins_ure A3 bei Kalluskulturen von Daucus carota. Z. Pflanzenphysiol., 68: 259-265.

Souer, E., Quattrocchio, F., de Vetten, N., Mol, J., Koes, R., 1995. A general method to isolate genes tagged by a high copy number transposable element. Plant J., 7: 677 -685 .

Sugano, N., Hayashi, K., 1967. Dynamic interrelation of cellular ingredients relevant to the biosynthesis of anthocyanin during tissue culture of carrot aggregen. Studies on anthocyanins LVII. Bot. Mag.,Tokyo, 80: 440- 449.

Tsuei, D. J., Chen, P. J., Lai, M. Y., Chen, D. S., Yang, C. S., Chen, J. Y., Hsu, T. Y., 1994. Inverse polymerase chain reaction for cloning cellular sequences adjacent to integrated hepatitis B virus DNA in hepatocellular carcinomas. J. Virol. Methods, 49: 269- 284.

Van den Broeck, D., Maes, T., Sauer, M., Zethof, J., De Keukeleire, P., D’Hauw, M., Van Montagu, M., Gerats, T., 1998. Transposon display identifies individual transposable elements in high copy number lines. Plant J., 13: $121-129$.

Walbot, V., 2000. Saturation mutagenesis using maize transposons. Curr. Opin. Plant Biol., 3: 103-107.

Yamamoto, Y., Mizuguchi, R., Yamada, Y., 1982. Selection of a high and stable pigment-producing strain in cultured Euphorbia millii cells. Theor. Appl. Genet., 61: $113-116$.

Yephremov, A., Saedler, H., 2000. Display and isolation of transposon-flanking sequences starting from genomic DNA or RNA. Plant J., 21: 495-505. 